\section{Prevención de la infección posquirúrgica en cirugía de terceras molares retenidas y semirretenidas utilizando dos regímenes de profilaxia antibiótica con Clindamicina}

Prevention of post surgical infection in retained and semi retained third molar surgery using two antibiotic prophylaxis regimens with Clindamycin

\section{Resumen}

El objetivo del estudio fue comparar dos esquemas de profilaxis antibiótica con clindamicina en pacientes sometidos a cirugía de tercera molar retenida o semirretenida. Se realizaron exodoncias de terceras molares, en sujetos sanos que cumplieron los criterios de inclusión, en la clínica de cirugía bucal y máxilofacial de la Facultad de Odontología de la UNMSM. El procedimiento quirúrgico se realizó empleando la técnica quirúrgica estándar, luego de realizada la exodoncia se programó citas de control durante los 5 días post extracción en donde se evaluó la aparición de signos de infección mediante la presencia de exudado o sangrado a nivel del lecho quirúrgico. Este registro se llevó a cabo para ambos grupos. Los datos obtenidos fueron analizados utilizando la prueba U de Mann-Whitney $(\mathrm{p}<0.05)$. No se encontró diferencia estadística significativa en la proporción de casos infectados entre los grupos. Sin embargo, los resultados muestran una menor cantidad de pacientes con infección en el grupo de la administración intramuscular $(10 \%)$ en relación al grupo de la administración oral (15\%). Se concluye que no existe diferencia significativa en la prevención de infecciones post quirúrgicas de terceras molares utilizando clindamicina por vía oral o por vía intramuscular.

\section{Abstract}

The aim of this study was to compare two antibiotic prophylaxis regimens with Clindamycin in patients under semi-retained third lower molar surgery. Third molar extractions were carried out in healthy patients who complied with the inclusion criteria, in the central clinic surgery service of San Marcos University dentistry faculty. After having performed the exodontia control appointments were programmed during 5 days post - extraction where the infection signs were evaluated through the presence or bleeding or exudates on the surgical bed. This registration was carried out for both groups. The obtained data was analyzed using the Mann-Whitney $U$ test $(\mathrm{p}<0.05)$.

A significant difference was not found in the proportion of cases infected among the groups. However, the results showed a smaller quantity of patients with infection in the group of the intramuscular via $(10 \%)$ in relation with the oral via group $(15 \%)$. It was concluded that there was not a significant difference in the prevention of post - surgical infections of third molars using clindamycin for oral or intramuscular via.

\section{Artículo Original}

\author{
Manuel Silva Infantes ${ }^{1}$, Miguel Rodrí- \\ guez Alfaro ${ }^{2}$, Antonio Cabrejos Álvarez ${ }^{1}$, \\ Jonny Burga Sánchez², Víctor Chumpitaz \\ Cerrate², Roger López Bellido², Arturo \\ Ramón Rosales ${ }^{2}$ Roberto Varas Hilario², \\ Juan Zegarra Cuya ${ }^{3}$ \\ Departamento Académico Médico \\ Quirúrgico \\ Departamento Académico de Ciencias \\ Básicas \\ Bachiller en Odontología \\ 1,2,3 Facultad Odontología, UNMSM
}

\section{Correspondencia:}

Mg. Manuel Silva Infantes. Facultad Odontología, UNMSM

Av. Germán Amézaga s/n, Lima, 1 Perú. e-mail: msilvai@unmsm.edu.pe

Palabras clave: Profilaxis antibiótica, clindamicina, tercera molar retenida.

Key words: Antibiotic prophylaxis clindamycin, retained third molar.

\section{Introducción}

La exodoncia de terceras molares retenidas es uno de los actos quirúrgicos que con mayor frecuencia se realiza en cirugía buco máxilofacial. Además, este procedimiento genera heridas quirúrgicas de tipo limpias contaminadas según la clasificación de Altemeier, por lo que se hace necesario indicar antibióticos de forma profiláctica ${ }^{1}$. La profilaxis antibiótica consiste en utilizar un antibiótico de manera preventiva que sea activo frente a las bacterias que suelen causar infecciones posteriores a los procedimientos quirúrgicos, y en mantener concentraciones tisulares eficaces durante toda la intervención quirúrgica.

Para ello, existen diferentes esquemas de profilaxis antibiótica. Sin embargo, son pocas las investigaciones que contrastan los diversos esquemas profilácticos. Estudios recientes señalan a la clindamicina como un antibiótico eficaz contra la flora bacteriana residente en boca y la flora patógena que con frecuencia infecta las heridas quirúrgicas.

La profilaxis antibiótica en cirugía, consiste en utilizar un antibiótico activo frente a las bacterias que con mayor frecuencia causan infecciones en la intervención en cuestión y en mantener concentraciones tisulares durante toda la intervención quirúrgica y el período posterior donde se puede producir una bacteriemia $^{1}$. Para lograr una adecuada profilaxis antibiótica, es necesario alcanzar niveles altos de antibióticos en los tejidos con riesgo de infección, por encima de la concentración inhibitoria mínima necesaria para inhibir el crecimiento del 90\% (CIM90) de bacterias patógenas de microorganismos pertinentes $^{2}$. Debe recalcarse que este 
procedimiento no elimina sino más bien disminuye el riesgo de infección de la herida operatoria. Cualquier procedimiento que lesione una mucosa donde hay un gran número de bacterias, como la mucosa bucofaríngea, producirá una bacteriemia transitoria generando que las bacterias puedan adherirse a la superficie de una válvula anormal o dañada con un peligro inminente de desarrollar una endocarditis bacteriana. ${ }^{3}$ Se ha sugerido que los procedimientos odontológicos tienen una participación mínima en el origen de endocarditis, si en realidad tienen alguna, cuestionando algunas de las recomendaciones para quimioprofilaxis; sin embargo, las recomendaciones persisten como el estándar de cuida$\mathrm{do}^{4}$. Estas complicaciones infecciosas de la herida operatoria también pueden presentarse en zonas distantes (metástasis sépticas por diseminación hematógeno-linfática), pero esto ocurre principalmente en pacientes inmunodeprimidos. ${ }^{5}$ Las directrices de la American Heart Association (AHA) para la prevención de la endocarditis bacteriana coinciden con la mayoría de los principios precedentes sobre una profilaxis eficaz. La endocarditis bacteriana, aunque es una enfermedad rara, tiene un riesgo de mortalidad elevado. ${ }^{6,8}$ Los métodos de profilaxis antibiótica incluyen fármacos que se pueden administrar por vía oral o parenteral en regímenes que no exceden por lo general las 24 horas. ${ }^{9,10}$

El objetivo de esta investigación fue comparar dos esquemas de administración profiláctica de clindamicina en pacientes sometidos a cirugía de tercera molar retenida o semirretenida.

\section{Material y método}

El estudio fue del tipo experimental, en el que participaron 40 pacientes de la Clínica de Cirugía Máxilo Facial de la Facultad de Odontología UNMSM, hombres y mujeres con una edad promedio de 25 años, sin antecedentes de enfermedad en el momento del estudio con diagnóstico de tercera molar retenida o semirretenida, sin presencia clínica de infección en curso y con indicación para exodoncia. Antes de ingresar a la investigación, cada uno de los integrantes de la muestra aceptó su participación a través de la firma del consentimiento informado. Los pacientes fueron distribuidos en dos grupos de veinte cada uno. Al primer grupo se le administró Clindamicina $600 \mathrm{mg}$ vía Oral (PO) una hora antes de la incisión, y al segundo grupo, Clindamicina $600 \mathrm{mg}$ por vía IM 30
Cuadro $\mathrm{N}^{\mathrm{o}} 1$. Frecuencias de pacientes según esquema de profilaxis antibiótica con clindamicina y desarrollo

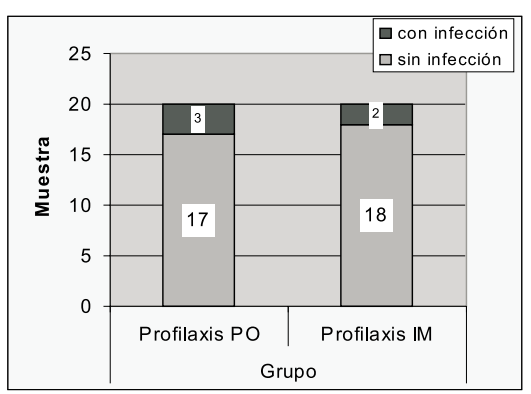

minutos antes de la incisión. Como terapia analgésica antiinflamatoria se le administró a todos los pacientes diclofenaco sódico $50 \mathrm{mg}$ + paracetamol $500 \mathrm{mg}$ (Dolocordralan extraforte ${ }^{\circledR}$ ) $\mathrm{PO}$ postoperatorio en regímenes de tres veces al día durante dos días.

Las exodoncias de terceras molares fueron realizadas por un mismo operador, en la Clínica de Cirugía Buco Máxilofacial de la Facultad de Odontología de la UNMSM durante los meses de junio - noviembre de 2005 y abril - octubre de 2006.

Luego de la cirugía, los participantes del estudio acudieron a la clínica durante los 5 días posextracción para realizar los controles respectivos en búsqueda de signos de infección del lecho quirúrgico: presencia de exudado o de sangrado. Si dentro de los 5 días después de realizada la extracción se presentaba signos de infección, el caso se consideraba como infectado; y si dentro de este lapso no presentaba los signos de infección descrito, el caso se consideraba no infectado.

\section{Resultados}

Las frecuencias de pacientes que presentaron infección posquirúrgica, se muestran en el Cuadro $\mathrm{N}^{\circ} 1$.

Según la Prueba U de Mann-Whitney $(p<0,05)$ no se encontró diferencia estadística significativa en la presencia de infección utilizando los esquemas de profilaxis con clindamicina por vía intramuscular y por vía oral $(\mathrm{p}$ $=1.00$ ).

\section{Discusión}

Los resultados obtenidos en el presente estudio demuestran que no existe diferencia estadísticamente significativa en la proporción de casos infectados entre los grupos, a pesar de que los resultados muestran una menor cantidad de pacientes con infección en el grupo de la administración intramuscular: $2(10 \%)$ en relación al grupo de la adminis- tración oral: 3 (15\%). Estos resultados concuerdan con los obtenidos por Lindeboom et al. ${ }^{2}$, quienes no encontraron diferencia significativa en profilaxis antibiótica usando clindamicina $600 \mathrm{mg}$ en dosis única y dosis de 24 horas, a pesar de que Ruggles et $\mathrm{al}^{11}$ manifiestan que el uso de antibióticos como profilaxis cerca del período preoperatorio, intraoperatorio y postoperatorio no brinda benéficos adicionales en la prevención de infecciones. Peterson $^{12}$ indica que sin profilaxis antibiótica la tasa de infección en heridas limpias contaminadas en cavidad oral varia desde $8 \%$ hasta un $25 \%$; lo que se asemeja con numerosos estudios que muestran diferencias estadísticamente significativas entre pacientes que recibieron profilaxis antibiótica frente a un control sin protección antibiótica. ${ }^{13,14,15}$ Otro punto crítico es la aparición de efectos adversos debido a la administración de antibióticos como la toxicidad, reacciones alérgicas, infecciones secundarias que pueden desarrollarse con una terapia antibiótica indiscriminada ${ }^{16}$; situaciones que pueden disminuir en incidencia al usar de forma correcta los antibióticos como profilaxis. Además, al elegir un régimen de profilaxis se requiere que el antibiótico muestre una adecuada penetración tisular como lo muestra clindamicina que posee una excelente penetración al tejido óseo y blando. ${ }^{17,18}$

La profilaxis antibiótica también ha demostrado ser efectiva en otros tipos de cirugía maxilofacial. Es el caso de un estudio prospectivo en pacientes con fractura mandibular en los que no se usaron antibióticos, la incidencia de infección que se reportó fue superior al $50 \%$, y el uso de profilaxis antibiótica mostró una reducción de la incidencia a menos del $6 \% .{ }^{19}$ Chole y Yee, ${ }^{20}$ en un estudio prospectivo aleatorio, realizado en 101 pacientes con fractura mandibular que fueron tratados con reducción abierta y cerrada; un grupo no recibió antibióticos y otro recibió 1 $\mathrm{g}$ de cefazolina endovenosa preoperatoria y postoperatoria, encontrando que la reducción de la incidencia de infección fue de 42 a 8,9\%.

Al término de la investigación se llegó a las siguientes conclusiones:

- Un alto porcentaje de pacientes que fueron sometidos a cirugía de terceras molares inferiores empleando clindamicina como profilaxis antibiótica por vía oral e intramuscular no presentaron infección en el postoperatorio. 
- No se encontró asociación entre la presencia de infección y el esquema de profilaxis antibiótica, empleado $600 \mathrm{mg}$ de clindamicina antes de la cirugía por vía oral e intramuscular.

- No se encontró diferencia estadística significativa en la prevención de infecciones postquirúrgicas empleando el esquema de profilaxis antibiótica con clindamicina por vía oral con respecto al esquema de profilaxis antibiótica con clindamicina por vía parenteral.

\section{Referencias bibliográficas}

1. Rodríguez M, Burga J, Chumpitaz V, Varas R, Guerra J, López R. Profilaxis Antibiótica en Estomatología. Odont Sanmarquina 2004; 8(1): 35-8.

2. Lindeboom J, Bass E, Kroon F. Prophylactic single-dose administration of $600 \mathrm{mg}$ clindamycin versus 4-time administration of $600 \mathrm{mg}$ clindamycin in orthognatic surgery: A prospective randomized study in bilateral mandibular sagittal ramus osteotomies. Oral Surg Oral Med Oral Pathol Oral Radiol Endod 2003; 95: 145-9.

3. Goodman and Gillman. Las bases farmacológicas de la terapéutica. 10 ed. México: editorial Mc Graw Hill - Interamericana; 2003: 1273-1275.

4. Strom B, Abrutyn E, Berlin J, Kinman J, Feldman R, Stolley P et al. Dental and cardiac risk factors for infective endocarditis. A population-based, cased-control study. Ann Intern Med 1998;129:761-9.
5. Cabrejos JA. Terapia antibiótica de la infección buco-facial odontogénica.

1 ed.Perú: Editorial e imprenta UNMSM; 2004:101-110.

6. Little JW, Falace DA. Tratamiento odontológico del paciente bajo tratamiento médico. 5 ed. Madrid (ES): Editorial Harcourt Brace; 1998.

7. Katzung BG. Farmacología Básica y Clínica. 8 ed. México: Editorial El Manual Moderno; 2002: 971-974.

8. Slavkin H. Uso adecuado de antibióticos en odontología. Quintessence (ed. esp.) 1999; 12(3): 182-199.

9. Lindeboom JAH, van der Akker. A prospective placebo-controlled doubleblind trial of antibiotic prophylaxis in intraoral bone grafting procedures: A pilot study. Oral Surg Oral Med Oral Pathol Oral radiol Endod 2003; 96: 669-72.

10. Abubaker A, Rollert M. Postoperative antibiotic prophylaxis in mandibular fractures: A preliminary ramdomized, double-blind, and placebo-controlled clinical study. J Oral Maxillofac Surg 2001; 59: 1415-1419.

11. Ruggles JE, Hann JR. Antibiotic prophylaxis in intraoral orthognatic surgery. J Oral Maxillofac Surg 1984;42:797-801

12. Peterson IJ. Antibiotic prophylaxis against wound infections in oral and maxillofacial surgery. J Oral Maxillofac Surg 1990;48:617-20.

13. Hotz G, Novotny-Lenhard J, Kinzig M, Soergel F. Single-dose antibiotic prophylaxis in maxillofacial surgery. Chemother 1994:40:65-9.

14. Zijderveld SA, Smeele LE, Kostense PJ, Tuinzing DB. Preoperative antibiotic prophylaxis in orthognatic surgery: A randomised, double blind, and placebo-controlled clinical study. J Oral Maxillofac Surg 1999;57:1403-6.

15. Piecuch JF, Arzadon J, Lieblich SE. Prophylactic antibiotics for third molar surgery. J Oral Maxillofac Surg 1995;53:53.

16. McHenry MC, Weinstein AJ. Antimicrobial drugs and infections in ambulatory patiens: Some problems and perspectives. Med Clin North Am 1983;67:3.

17. Mueller SC, Henkel KO, Neumann J. Perioperative antibiotic prophylaxis in maxillofacial surgery. Penetration of clindamicina in various tissues. J Craniomaxillofac Surg 1999;27:172.

18. Jauregui LE, Senour CL. Chronic osteomielitis, in Jáuregui LE (ed): Diagnosis and Management of Bone Infections. New York, NY, Marcel Dekker, 1995;pp37-108.

19. Zallen RD, Curry JT. A study of antibiotic usage in compound mandibular fractures. J Oral Surg 1975; 33: 431.

20. Chole RA, Yee J. Antibiotic prophylaxis for facial fractures. Arch Otoralyngol Head Neck Surg 1987; 113: 1055.

Recibido : 26-04-2007

Aceptado para publicación: 25-05-2007 